\title{
Efeito de diferentes substratos e adubação fosfatada no crescimento de mudas de Rhapis excelsa (Thunb.) A. Henry ex Rehder (Palmeira-ráfia) ${ }^{(1)}$
}

\author{
PETTERSON BAPTISTA DA LUZ ${ }^{(2)}$, PATRICIA DUARTE DE OLIVEIRA PAIVA ${ }^{(3)}$, ARMANDO REIS TAVARES ${ }^{(4)}$, \\ SHOEY KANASHIRO(4), FRANCISMAR FRANCISCO ALVES AGUIAR(4)
}

\begin{abstract}
RESUMO
Palmeira-ráfia (Rhapis excelsa (Thunb.) A. Henry ex Rehder) é uma das palmeiras ornamentais mais cultivadas em todo mundo, indicada principalmente para vasos e áreas de sombra. O objetivo deste estudo foi investigar o efeito de substratos no crescimento de mudas da palmeira-ráfia. Foram utilizadas 240 plantas com aproximadamente um ano de idade, plantadas em sacos de polietileno de 2,5 litros, contendo os diferentes tratamentos. Para a composição dos substratos, foram utilizados terra, esterco de curral, areia e casca de arroz carbonizada, em todas as combinações possíveis, nas mesmas proporções, com e sem a aplicação de superfosfato simples ( $10 \mathrm{~g} \mathrm{~L}^{-1}$ substrato), totalizando 30 tratamentos. O delineamento foi inteiramente casualizado, com 4 repetições e duas plantas por parcela. As variáveis analisadas foram altura da planta, número de folhas, diâmetro do caule e número de brotações. Verificou-se para todos os parâmetros a superioridade do substrato constituído por esterco, em relação aos demais. Os substratos constituídos por 1 terra +1 esterco +1 areia, 1 terra +1 esterco +1 casca de arroz carbonizada e 1 terra +1 esterco +1 areia +1 casca de arroz carbonizada proporcionaram a formação de mudas com qualidade equivalente ao substrato constituído por apenas esterco. Não houve resposta da $R$. excelsa à adubação fosfatada.
\end{abstract}

Palavras-chave: planta ornamental, palmeira, substrato.

\section{ABSTRACT}

Effect of different substrates for seedlings development Rhapis excelsa (Thunb.) A. Henry ex Rehder (Lady palm)

Lady palm (Rhapis excelsa (Thunb.) A. Henry ex Rehder) is an ornamental palm widely cultivated worldwide, specially indicated for shaded areas and as vases plant. The purpose of this research was to investigate the effect of substrates on lady palm seedlings growth. One year-old seedlings (240) produced by seeds were transplanted to polyethylene bags containing 2.5 liters of substrate. Thirty treatments, involving four substrates composed with soil, manure, sand and carbonized rice husk in all combinations and same proportions and/or 10 grams $\mathrm{L}^{-1}$ of superphosphate were used. The experimental design was randomized with four replicates with two plants per replicate. The analyzed parameters were seedlings height, number of leaves, stem diameter and number of shoots. The substrate composed only with manure showed a better performance for all parameters evaluated. Seedlings cultivated in substrates composed with 1 soil +1 manure +1 sand, 1 soil +1 manure +1 carbonized rice husk and 1 soil +1 manure +1 sand +1 carbonized rice husk presented an equivalent quality as those cultivated in manure only. Fertilization with simple superphosphate did not promote lady palm seedlings growth.

Keywords: ornamental plant, palms, substrate.

\section{INTRODUÇÃO}

A palmeira Rhapis excelsa, nativa do Sul da China, pertence à família Arecaceae, sendo popularmente denominada de palmeira-senhora ou palmeira-ráfia (McCURRACH, 1960; BLOMBERY e ROOD, 1982; DOMINGUES, 1995). A espécie apresenta caules múltiplos, cobertos com fibras marrons emaranhadas, formando touceiras, que podem alcançar 4,5 metros de altura, as folhas são palmadas, divididas até a base em 5-9 segmentos irregulares (LORENZI et al., 2004).

A espécie possui alto valor ornamental por ser pouco exigente em relação à luminosidade, tolerando a baixa luminosidade na fase jovem até ambientes a pleno sol, quando adulta, podendo ser cultivada em vasos e ambientes fecha- dos. A multiplicação dessa espécie é realizada por meio de sementes ou divisão de touceiras (LORENZI et al., 2004).

Poucas referências existem relativas a estudos e recomendações de substratos para o cultivo de palmeiras. Entre estas referências, podem-se citar BOVI (1994), que utilizou como substrato para cultivo da palmeira Euterpe oleracea Mart. (açaí), mistura composta de solo (latossolo roxo), com resíduos de algodão decomposto, nas proporções de 25 e 50\%; AGUIAR et al. (1996), que utilizaram como substrato para cultivo da palmeira Geonoma schottiana Mart., mistura contendo terra vegetal e esterco bovino, na proporção de 1:1; AGUIAR et al. (2002), que, em experimento com a palmeira Euterpe edulis Mart., recomendam como substrato uma mistura de terra (latossolo vermelho amarelo) e serapilheira, em partes iguais; e BROSCHAT e MEE-

\footnotetext{
(1) Recebido para publicação em 24/06/2009 e aceito em 20/05/2011.

(2) Prof. Doutor, Departamento de Agronomia, Universidade do Estado do Mato Grosso/Unemat, Campus Universitário de Cáceres, Avenida São João, s/n Cavalhada 78200-000. Cáceres, MT. E-mail: petterbaptista@yahoo.com.br.

(3) Profa. Doutora, Departamento de Agricultura, Universidade Federal de Lavras (UFLA) 37200-000, Lavras, MG.

(4) Pesquisador Científico, Seção de Ornamentais Instituto de Botânica Secretaria do Meio Ambiente, SP Cx. P. 4005 - 01061-970 São Paulo, SP.
} 
ROW (1990), que, para a produção de mudas de palmeiras em recipientes, sugerem um substrato com maior fração de areia.

A palmeira $R$. excelsa se adapta bem a diversos tipos de solo, mas seu melhor desenvolvimento ocorre em solos de boa drenagem, ricos em húmus (matéria orgânica) e $\mathrm{pH}$ entre 5,5 e 7,0 (McKAMEY, 1989). DEMATTÊ et al. (1994) recomendam para cultivo de palmeira $R$. excelsa (palmeira-ráfia) substrato constituído de terra, esterco bovino e vermiculita, na proporção de 2:1:1. LUZ et al. (2008) utilizaram como substrato para o cultivo da mesma palmeira, a mistura de terra, esterco e areia (1:1:1), sem adubação complementar.

O presente trabalho objetivou avaliar o efeito de diferentes tipos e composição de substratos e adubação fosfatada no desenvolvimento de mudas de palmeira-ráfia (Rhapis excelsa (Thunberg) Henry ex. Rehder).

\section{MATERIAL E MÉTODOS}

O experimento foi realizado em telado (50\% de sombreamento) do Departamento de Agricultura, na Universidade Federal de Lavras (Lavras-MG). As coordenadas geográficas são $21^{\circ} 14^{\prime} 30^{\prime \prime}$ de latitude Sul e $45^{\circ} 00^{\prime} 10^{\prime \prime}$ de longitude oeste, altitude de 918 metros e precipitação média anual de 1.529,7 mm (BRASIL, 1992), com clima, segundo Koppen, tipo Cwa com características $\mathrm{Cwb}$, apresentando duas estações bem definidas: seca, com temperaturas mais baixas de abril a setembro e chuvosa, com temperaturas mais elevadas de outubro a março. O estudo foi conduzido de abril de 2003 a abril de 2004.

Foram utilizadas 240 plantas, com aproximadamente um ano de idade, produzidas a partir de sementes no Instituto de Botânica, São Paulo, SP. As mudas foram selecionadas e transplantadas para sacos de polietileno com capacidade para 2,5 L e acondicionadas sob telado com 50\% de sombreamento e sistema de irrigação por microaspersão. Os tratamentos foram constituídos de diferentes substratos, utilizando, como constituintes, terra, esterco bovino, areia e casca de arroz carbonizada, formulados em todas as combinações possíveis e acrescidos ou não do adubo superfosfato simples na dosagem de 10 gramas por litro de substrato. As formulações dos substratos foram assim denominadas: substratos 1 (terra-T), 2 (esterco bovino curtido-E), 3 (areiaA), 4 (casca de arroz carbonizada-CAC), $5(\mathrm{~T}+\mathrm{E}), 6(\mathrm{~T}+$ $\mathrm{A}), 7(\mathrm{~T}+\mathrm{CAC}), 8(\mathrm{E}+\mathrm{A}), 9(\mathrm{E}+\mathrm{CAC}), 10(\mathrm{~A}+\mathrm{CAC})$, $11(\mathrm{~T}+\mathrm{E}+\mathrm{A}), 12(\mathrm{~T}+\mathrm{E}+\mathrm{CAC}), 13(\mathrm{~T}+\mathrm{A}+\mathrm{CAC}), 14$ $(\mathrm{E}+\mathrm{A}+\mathrm{CAC})$ e $15(\mathrm{~T}+\mathrm{E}+\mathrm{A}+\mathrm{CAC}) . \mathrm{O}$ delineamento experimental foi em esquema fatorial 15 (formulações de substratos) x 2 (adição ou não de superfosfato), totalizando 30 tratamentos com 4 repetições de 2 plantas por parcela, sendo cultivada uma planta por saco de polietileno.

O experimento foi conduzido no período de 360 dias, tendo sido as avaliações realizadas em intervalos de três meses após a instalação do experimento. Os parâmetros avaliados foram altura da planta medida do nível do substrato à primeira inserção de folha $(\mathrm{cm})$, diâmetro do caule no nível do substrato $(\mathrm{cm})$ e número de folhas e de brotações.

As análises químicas dos substratos foram realizadas no Laboratório de Nutrição Mineral de Plantas do Departamento de Ciências do Solo da Universidade Federal de Lavras.
A metodologia para a realização das análises foi de acordo com SONNEVELD et al. (1974), sendo que os substratos analisados foram todas as formulações (15) sem a adição de fósforo. Pela análise química dos substratos, podem-se identificar as características químicas entre as diferentes formulações (Tabela 1).

Os resultados foram submetidos à análise de variância com o programa SISVAR ${ }^{\circledR}$ (FERREIRA, 2000), utilizando o teste de Scott Knott a 5\% de probabilidade para a comparação entre os tratamentos.

\section{RESULTADOS E DISCUSSÃO}

O parâmetro altura das plantas apresentou interação significativa entre o substrato e a adubação na primeira, terceira e quarta avaliações. Foram realizados os desdobramentos para as duas variáveis analisadas (substrato e adubação) e as médias encontram-se na Tabela 2.

Na primeira avaliação, realizada três meses após a instalação dos experimentos, as alturas médias variaram de 1,46 a 2,09 $\mathrm{cm}$ para os substratos que se destacaram na primeira avaliação sem adição de adubo, e de 1,29 a 1,69 cm para os substratos acrescidos de adubo. Em relação à aplicação de adubação, foram observadas diferenças entre os substratos $1(\mathrm{~T}), 9(\mathrm{E}+\mathrm{CAC}), 13(\mathrm{~T}+\mathrm{A}+\mathrm{CAC})$ e $15(\mathrm{~T}+\mathrm{E}+\mathrm{A}+$ $\mathrm{CAC})$, que apresentaram maiores alturas das plantas quando acrescidos do adubo, tendo sido estas diferenças acentuadas na última avaliação para os substratos 1 e 6 .

A partir dos seis meses, os substratos apresentaram maiores diferenças na altura das plantas, havendo maior crescimento das plantas cultivadas em substratos acrescidos de esterco. Segundo CUNHA et al. (2006), na composição do substrato para o crescimento de plântulas, a fonte orgânica é responsável pela retenção de umidade e fornecimento de parte dos nutrientes. Aos nove e 12 meses, além do substrato esterco, os substratos $5(\mathrm{~T}+\mathrm{E}), 7(\mathrm{~T}+\mathrm{CAC}), 8$ $(\mathrm{E}+\mathrm{A}), 9(\mathrm{E}+\mathrm{CAC}), 11(\mathrm{~T}+\mathrm{E}+\mathrm{A}), 12(\mathrm{~T}+\mathrm{E}+\mathrm{CAC})$, $13(\mathrm{~T}+\mathrm{A}+\mathrm{CAC}), 14(\mathrm{E}+\mathrm{A}+\mathrm{CAC})$ e $15(\mathrm{~T}+\mathrm{E}+\mathrm{A}+$ CAC) também proporcionaram incremento na altura das mudas. Verifica-se que os substratos que apresentam na sua composição CAC e E, usados isoladamente ou associados, proporcionam um bom crescimento de mudas, mesmo sem a adição de adubo.

O número de folhas também apresentou interação significativa entre os substratos e a adubação fosfatada, em todas as datas de coletas. Foram realizados os desdobramentos para os dois itens testados: substrato e adubação. As médias observadas para o número de folhas formadas em função dos tratamentos testados encontram-se na Tabela 3.

Os substratos que continham esterco bovino influenciaram, satisfatoriamente, no desenvolvimento de folhas da palmeira-ráfia. Na primeira avaliação, os substratos $2(\mathrm{E}), 8$ $(\mathrm{E}+\mathrm{A}), 9(\mathrm{E}+\mathrm{CAC})$ e $11(\mathrm{~T}+\mathrm{E}+\mathrm{A})$ sem adição de adubação e o substrato 2 com adubação se diferenciaram e foram estatisticamente superiores aos demais, proporcionando formação de maior número de folhas (Tabela 3). Entretanto, comparando a aplicação de adubação fosfatada ou não entre os substratos, não houve diferença significativa nessa fase. Na segunda análise, realizada aos seis meses, os substratos 2 (E) e $8(E+A)$ proporcionaram formação de maior número de folhas em relação aos demais tratamentos. Na terceira 
Tabela 1. Características químicas dos substratos utilizados na palmeira-ráfia.

Table 1. Chemical characteristics of substrate used on Lady-palm.

\begin{tabular}{|c|c|c|c|c|c|c|c|c|}
\hline \multirow[b]{2}{*}{ Substratos } & \multicolumn{8}{|c|}{ Macronutrientes $\left(\mathrm{mg.dm}^{-3}\right)$} \\
\hline & ${\mathrm{N}-\mathrm{NH}_{4}^{+}}^{+}$ & $\mathrm{N}^{-\mathrm{NO}_{3}^{-}}$ & $\mathbf{P}$ & $\mathbf{K}$ & $\mathbf{N a}$ & $\mathbf{C a}$ & Mg & $\mathbf{S}$ \\
\hline 1 & 4,5 & 183,0 & 7,40 & 161,32 & 31,5 & 42,6 & 379,9 & 32,6 \\
\hline 2 & 4,5 & 184,5 & 15,07 & 220,52 & 30,0 & 31,9 & 318,6 & 53,9 \\
\hline 3 & 4,5 & 21,0 & 3,56 & 32,56 & 6,0 & 2,3 & 17,1 & 50,5 \\
\hline 4 & 4,5 & 19,5 & 0,19 & 51,80 & 9,0 & 10,0 & 30,8 & 32,6 \\
\hline 5 & 4,5 & 16,5 & 0,10 & 20,72 & 7,5 & 13,8 & 30,9 & 22,2 \\
\hline 6 & 4,5 & 102,0 & 0,10 & 164,28 & 33,0 & 105,0 & 555,2 & 103,4 \\
\hline 7 & 4,5 & 18,0 & 0,10 & 25,16 & 7,5 & 19,6 & 50,3 & 35,4 \\
\hline 8 & 4,5 & 34,5 & 1,44 & 56,24 & 6,0 & 1,8 & 7,95 & 10,9 \\
\hline 9 & 4,5 & 6,0 & 0,14 & 2,52 & 3,0 & 1,7 & 5,6 & 0,9 \\
\hline 10 & 4,5 & 30,0 & 13,20 & 272,32 & 48,0 & 69,2 & 623,4 & 53,9 \\
\hline 11 & 4,5 & 129,0 & 0,64 & 173,16 & 27,0 & 36,4 & 312,0 & 65,0 \\
\hline 12 & 4,5 & 129,0 & 0,33 & 118,40 & 27,0 & 40,9 & 289,1 & 57,9 \\
\hline 13 & 4,5 & 117,0 & 0,59 & 109,52 & 19,5 & 25,5 & 176,8 & 35,4 \\
\hline 14 & 4,5 & 138,0 & 10,80 & 145,04 & 25,5 & 21,8 & 195,6 & 30,9 \\
\hline 15 & 4,5 & 16,5 & 0,14 & 45,88 & 57,0 & 8,1 & 22,41 & 18,1 \\
\hline
\end{tabular}

\begin{tabular}{|c|c|c|c|c|c|c|c|}
\hline \multirow[b]{2}{*}{ Substratos } & \multicolumn{5}{|c|}{ Micronutrientes } & \multirow[b]{2}{*}{ pH $\mathrm{H}_{2} \mathrm{O}(1: 1,5)$} & \multirow[b]{2}{*}{$\mathrm{CE}\left(\mathrm{mS} . \mathrm{cm}^{-1}\right)$} \\
\hline & B $\quad\left(\mathrm{mg} . \mathrm{dm}^{-3}\right)$ & $\mathrm{Cu}\left(\mu \mathrm{g} . \mathrm{dm}^{-3}\right)$ & $\mathrm{Fe} \quad\left(\mathrm{mg} \cdot \mathrm{dm}^{-3}\right)$ & $\operatorname{Mn}\left(\mu \mathrm{g} . \mathrm{dm}^{-3}\right)$ & $\mathrm{Zn}\left(\mu \mathrm{g} . \mathrm{dm}^{-3}\right)$ & & \\
\hline 1 & 0,42 & 21,0 & 0,77 & 0,0 & 0,0 & 6,79 & 1,56 \\
\hline 2 & 0,45 & 30,0 & 2,74 & 16,5 & 19,5 & 6,94 & 1,52 \\
\hline 3 & 0,25 & 3,0 & 20,12 & 342,0 & 9,0 & 7,58 & 0,14 \\
\hline 4 & 0,20 & 0,0 & 0,29 & 3,0 & 0,0 & 7,16 & 0,31 \\
\hline 5 & 0,15 & 0,0 & 0,0 & 0,0 & 0,0 & 7,12 & 0,24 \\
\hline 6 & 0,64 & 0,0 & 0,0 & 3,0 & 0,0 & 6,74 & 2,09 \\
\hline 7 & 0,20 & 0,0 & 0,0 & 0,0 & 0,0 & 7,17 & 0,28 \\
\hline 8 & 0,20 & 0,0 & 0,86 & 33,0 & 0,0 & 7,25 & 0,24 \\
\hline 9 & 0,13 & 0,0 & 0,43 & 766,5 & 1,5 & 7,15 & 0,63 \\
\hline 10 & 0,47 & 43,5 & 0,66 & 111,0 & 37,5 & 7,14 & 2,80 \\
\hline 11 & 0,32 & 9,0 & 0,06 & 96,0 & 0,0 & 6,90 & 2,02 \\
\hline 12 & 0,27 & 4,5 & 0,22 & 231,0 & 9,0 & 7,57 & 1,74 \\
\hline 13 & 0,30 & 1,5 & 0,21 & 66,0 & 0,0 & 6,87 & 1,25 \\
\hline 14 & 0,20 & 33,0 & 3,09 & 1141,5 & 141,0 & 7,20 & 1,63 \\
\hline 15 & 0,13 & 10,5 & 0,49 & 402,0 & 669,0 & 7,25 & 0,84 \\
\hline
\end{tabular}

Substratos: 1 (terra-T), 2 (esterco-E), 3 (areia-A), 4 (casca de arroz carbonizada-CAC), 5 (T + E), 6 (T + A), 7 (T + CAC), 8 (E + A), 9 (E + CAC), $10(\mathrm{~A}+\mathrm{CAC}), 11(\mathrm{~T}+\mathrm{E}+\mathrm{A}), 12(\mathrm{~T}+\mathrm{E}+\mathrm{CAC}), 13(\mathrm{~T}+\mathrm{A}+\mathrm{CAC}), 14(\mathrm{E}+\mathrm{A}+\mathrm{CAC}) \mathrm{e} 15(\mathrm{~T}+\mathrm{E}+\mathrm{A}+\mathrm{CAC})$.

Substrates: 1 (soil-T), 2 (manure-E), 3 (sand-A), 4 (carbonized rice husk-CAC), $5(T+E), 6(T+A), 7(T+C A C), 8(E+A), 9(E+C A C), 10(A$

$+C A C), 11(T+E+A), 12(T+E+C A C), 13(T+A+C A C), 14(E+A+C A C)$ e $15(T+E+A+C A C)$. 
Tabela 2. Médias de altura (cm) de palmeira-ráfia cultivadas em diferentes substratos, com e sem adição de adubação fosfatada. Avaliações: $3\left(1^{\mathrm{a}}\right), 6\left(2^{\mathrm{a}}\right), 9\left(3^{\mathrm{a}}\right)$ e12 (4 $\left.4^{\mathrm{a}}\right)$ meses.

Table 2. Means values of Lady-palm cultivated in different substrates with or without phosphate fertilization. Evaluations: 3 (1st); 6 (2nd); 9 (3rd) and 12 (4th) mon.

\begin{tabular}{|c|c|c|c|c|c|c|c|}
\hline \multirow{3}{*}{ Substrato } & \multirow{2}{*}{\multicolumn{2}{|c|}{$\frac{1^{\mathrm{a}} \text { avaliação }}{\text { Adubação }}$}} & \multirow{3}{*}{$2^{\mathrm{a}}$ avaliação } & \multirow{2}{*}{\multicolumn{2}{|c|}{$\begin{array}{c}3^{\mathrm{a}} \text { avaliação } \\
\text { Adubação }\end{array}$}} & \multirow{2}{*}{\multicolumn{2}{|c|}{$\frac{4^{\mathrm{a}} \text { avaliação }}{\text { Adubação }}$}} \\
\hline & & & & & & & \\
\hline & Com & Sem & & Com & Sem & Com & Sem \\
\hline 1 & 1,54 a $\mathrm{A}$ & $0,90 \mathrm{~b} \mathrm{~B}$ & $1,82 \mathrm{c}$ & 3,27 a A & $1,34 \mathrm{c} \mathrm{B}$ & 4,11 a $\mathrm{A}$ & 0,92 c B \\
\hline 2 & 1,61 a $\mathrm{A}$ & 2,09 a A & $3,57 \mathrm{a}$ & 4,17 a $A$ & 4,79 a $\mathrm{A}$ & 5,84 a $\mathrm{A}$ & 6,29 a A \\
\hline 3 & $1,07 \mathrm{~b} \mathrm{~A}$ & $1,04 \mathrm{~b} \mathrm{~A}$ & $1,45 \mathrm{c}$ & $1,46 \mathrm{~b} \mathrm{~A}$ & $1,66 \mathrm{c} \mathrm{A}$ & $2,24 \mathrm{~b} \mathrm{~A}$ & $2,05 \mathrm{c} \mathrm{A}$ \\
\hline 4 & $0,89 \mathrm{~b} \mathrm{~A}$ & $0,89 \mathrm{~b} \mathrm{~A}$ & $1,44 \mathrm{c}$ & $1,95 \mathrm{~b} \mathrm{~A}$ & $2,09 \mathrm{~b} \mathrm{~A}$ & $2,95 \mathrm{~b} \mathrm{~A}$ & $2,81 \mathrm{~b} \mathrm{~A}$ \\
\hline 5 & 1,29 a A & 1,46 a $\mathrm{A}$ & $2,36 \mathrm{~b}$ & 3,92 a A & 3,79 a $\mathrm{A}$ & 5,45 a $\mathrm{A}$ & 5,22 a A \\
\hline 6 & $0,74 \mathrm{~b} \mathrm{~A}$ & $0,92 \mathrm{~b} \mathrm{~A}$ & $1,33 \mathrm{c}$ & $2,25 \mathrm{~b} \mathrm{~A}$ & $1,15 \mathrm{c} \mathrm{B}$ & $3,24 \mathrm{~b} \mathrm{~A}$ & 1,87 c B \\
\hline 7 & 1,50 a $\mathrm{A}$ & $1,26 \mathrm{~b} \mathrm{~A}$ & $1,93 \mathrm{c}$ & 3,42 a A & $2,31 \mathrm{~b} \mathrm{~B}$ & 4,46 a $\mathrm{A}$ & $3,44 \mathrm{~b} \mathrm{~A}$ \\
\hline 8 & 1,35 a $\mathrm{A}$ & $0,89 \mathrm{~b} \mathrm{~A}$ & $2,58 \mathrm{~b}$ & 3,66 a $A$ & 3,02 a A & 5,00 a $\mathrm{A}$ & 4,70 a $\mathrm{A}$ \\
\hline 9 & 1,15 b B & 1,76 a $\mathrm{A}$ & $2,61 \mathrm{~b}$ & 3,39 a $A$ & 3,46 a $\mathrm{A}$ & $3,56 \mathrm{~b} \mathrm{~A}$ & 5,67 a $A$ \\
\hline 10 & $1,02 \mathrm{~b} \mathrm{~A}$ & $1,05 \mathrm{~b} \mathrm{~A}$ & $1,59 \mathrm{c}$ & $2,04 \mathrm{~b} \mathrm{~A}$ & $2,34 \mathrm{~b} \mathrm{~A}$ & $2,65 \mathrm{~b} \mathrm{~A}$ & $3,41 \mathrm{~b} \mathrm{~A}$ \\
\hline 11 & 1,42 a $\mathrm{A}$ & $0,96 \mathrm{~b} \mathrm{~A}$ & $2,62 \mathrm{~b}$ & 4,20 a A & 4,05 a A & 5,39 a $A$ & 5,11 a $A$ \\
\hline 12 & $1,09 \mathrm{~b} \mathrm{~A}$ & 1,85 a A & $2,69 \mathrm{~b}$ & 3,56 a $\mathrm{A}$ & 3,92 a $A$ & 5,15 a A & 5,40 a $A$ \\
\hline 13 & $1,06 \mathrm{~b} \mathrm{~A}$ & $0,92 \mathrm{~b} B$ & $1,74 \mathrm{c}$ & 3,18 a A & $2,31 \mathrm{~b} \mathrm{~A}$ & 4,20 a A & $3,39 \mathrm{~b} \mathrm{~A}$ \\
\hline 14 & 1,69 a $\mathrm{A}$ & $1,26 \mathrm{~b} \mathrm{~A}$ & $2,67 \mathrm{~b}$ & 3,39 a $A$ & 3,80 a $\mathrm{A}$ & 4,71 a $\mathrm{A}$ & 4,39 a $A$ \\
\hline 15 & $1,11 \mathrm{~b} \mathrm{~B}$ & 1,81 a $\mathrm{A}$ & $2,65 \mathrm{~b}$ & 3,50 a A & 3,95 a $\mathrm{A}$ & 4,82 a A & 5,42 a $A$ \\
\hline
\end{tabular}

*Médias seguidas da mesma letra minúscula na coluna (substrato) e maiúsculas nas linhas (adubação), não diferem entre si a 5\% de probabilidade pelo teste de Scott-Knott.

Substratos: 1 (terra-T), 2 (esterco-E), 3 (areia-A), 4 (casca de arroz carbonizada-CAC), $5(\mathrm{~T}+\mathrm{E}), 6(\mathrm{~T}+\mathrm{A}), 7(\mathrm{~T}+\mathrm{CAC}), 8(\mathrm{E}+\mathrm{A}), 9(\mathrm{E}+\mathrm{CAC}), 10$ $(\mathrm{A}+\mathrm{CAC}), 11(\mathrm{~T}+\mathrm{E}+\mathrm{A}), 12(\mathrm{~T}+\mathrm{E}+\mathrm{CAC}), 13(\mathrm{~T}+\mathrm{A}+\mathrm{CAC}), 14(\mathrm{E}+\mathrm{A}+\mathrm{CAC}) \mathrm{e} 15(\mathrm{~T}+\mathrm{E}+\mathrm{A}+\mathrm{CAC})$.

*Means followed by the same small letter in columns (substrate) and capital letter in lines (fertilization) did not differ by Scott-Knott test at $5 \%$.

Substrates: 1 (soil-T), 2 (manure-E), 3 (sand-A), 4 (carbonized rice husk-CAC), 5 (T + E), 6 (T + A), 7 (T + CAC), 8 (E + A), 9 (E + CAC), 10 (A + $\mathrm{CAC}), 11(\mathrm{~T}+\mathrm{E}+\mathrm{A}), 12(\mathrm{~T}+\mathrm{E}+\mathrm{CAC}), 13(\mathrm{~T}+\mathrm{A}+\mathrm{CAC}), 14(\mathrm{E}+\mathrm{A}+\mathrm{CAC})$ e $15(\mathrm{~T}+\mathrm{E}+\mathrm{A}+\mathrm{CAC})$.

Tabela 3. Médias de número de folhas de palmeira ráfia cultivadas em diferentes substratos com e sem adição de adubação fosfatada. Avaliações: aos $3\left(1^{\mathrm{a}}\right), 6\left(2^{\mathrm{a}}\right), 9\left(3^{\mathrm{a}}\right)$ e12 (4a) meses.

Table 3. Means values of number of leaves Lady-palm cultivated in different substrates with or without phosphate fertilization. Evaluations: 3 (1st); 6 (2nd); 9 (3rd) and 12 (4th) month.

\begin{tabular}{|c|c|c|c|c|c|c|c|c|}
\hline \multirow{3}{*}{ Substrato } & \multirow{2}{*}{\multicolumn{2}{|c|}{$\begin{array}{c}1^{\mathrm{a}} \text { avaliação } \\
\text { Adubação }\end{array}$}} & \multirow{2}{*}{\multicolumn{2}{|c|}{$\begin{array}{c}2^{a} \text { avaliação } \\
\text { Adubação }\end{array}$}} & \multirow{2}{*}{\multicolumn{2}{|c|}{$\begin{array}{c}3^{\mathrm{a}} \text { avaliação } \\
\text { Adubação }\end{array}$}} & \multirow{2}{*}{\multicolumn{2}{|c|}{$\begin{array}{c}4^{\mathrm{a}} \text { avaliação } \\
\text { Adubação }\end{array}$}} \\
\hline & & & & & & & & \\
\hline & Com & Sem & Com & Sem & Com & Sem & Com & Sem \\
\hline 1 & $3,87 \mathrm{bA}$ & $2,25 \mathrm{~dB}$ & $5,12 \mathrm{bA}$ & $2,25 \mathrm{~dB}$ & $5,75 \mathrm{bA}$ & $2,75 \mathrm{~dB}$ & $6,12 \mathrm{aA}$ & $2,37 \mathrm{~dB}$ \\
\hline 2 & $4,50 \mathrm{aA}$ & $4,50 \mathrm{aA}$ & $6,12 \mathrm{aA}$ & $6,12 \mathrm{aA}$ & $7,25 \mathrm{aA}$ & $7,87 \mathrm{aA}$ & $7,37 \mathrm{aA}$ & $7,25 \mathrm{aA}$ \\
\hline 3 & $3,00 \mathrm{cA}$ & $2,75 \mathrm{cA}$ & $3,87 \mathrm{dA}$ & $3,62 \mathrm{cA}$ & $4,75 \mathrm{cA}$ & $4,50 \mathrm{cA}$ & $5,00 \mathrm{bA}$ & $4,75 \mathrm{cA}$ \\
\hline 4 & $2,75 \mathrm{cA}$ & $3,12 \mathrm{cA}$ & $3,75 \mathrm{dA}$ & $4,12 \mathrm{cA}$ & $4,62 \mathrm{cA}$ & $5,00 \mathrm{cA}$ & $5,12 \mathrm{bA}$ & $6,12 \mathrm{bA}$ \\
\hline 5 & $3,62 \mathrm{bA}$ & $3,62 \mathrm{bA}$ & $5,12 \mathrm{bA}$ & $5,00 \mathrm{bA}$ & $6,12 \mathrm{bA}$ & $6,50 \mathrm{bA}$ & $6,87 \mathrm{aA}$ & $6,87 \mathrm{aA}$ \\
\hline 6 & $3,12 \mathrm{cA}$ & $3,00 \mathrm{cA}$ & $4,25 \mathrm{cA}$ & $3,87 \mathrm{cA}$ & $4,87 \mathrm{cA}$ & $4,62 \mathrm{cA}$ & $5,12 \mathrm{bA}$ & $4,62 \mathrm{cA}$ \\
\hline 7 & $3,00 \mathrm{cA}$ & $3,25 \mathrm{cA}$ & $4,62 \mathrm{cA}$ & $4,12 \mathrm{cA}$ & $5,87 \mathrm{bA}$ & $5,25 \mathrm{cA}$ & $6,37 \mathrm{aA}$ & $5,87 \mathrm{bA}$ \\
\hline 8 & $4,00 \mathrm{bA}$ & $4,12 \mathrm{aA}$ & $5,75 \mathrm{aA}$ & $5,37 \mathrm{bA}$ & $7,00 \mathrm{aA}$ & $6,62 \mathrm{bA}$ & $7,00 \mathrm{aA}$ & $6,37 \mathrm{bA}$ \\
\hline 9 & $3,50 \mathrm{bA}$ & $4,00 \mathrm{aA}$ & $4,62 \mathrm{cB}$ & $5,50 \mathrm{bA}$ & $6,25 \mathrm{bA}$ & $6,75 \mathrm{bA}$ & $5,50 \mathrm{bB}$ & $7,62 \mathrm{aA}$ \\
\hline 10 & $2,75 \mathrm{cA}$ & $3,00 \mathrm{cA}$ & $3,62 \mathrm{dA}$ & $4,12 \mathrm{cA}$ & $4,50 \mathrm{cA}$ & $5,12 \mathrm{cA}$ & $4,62 \mathrm{bA}$ & $5,75 \mathrm{bA}$ \\
\hline 11 & $3,75 \mathrm{bA}$ & $4,00 \mathrm{aA}$ & $5,50 \mathrm{bA}$ & $5,37 \mathrm{bA}$ & $6,87 \mathrm{aA}$ & $6,50 \mathrm{bA}$ & $6,75 \mathrm{aA}$ & $7,12 \mathrm{aA}$ \\
\hline 12 & $3,50 \mathrm{bA}$ & $3,62 \mathrm{bA}$ & $5,12 \mathrm{bA}$ & $5,00 \mathrm{bA}$ & $6,37 \mathrm{bA}$ & $6,37 \mathrm{bA}$ & $6,62 \mathrm{aA}$ & $7,25 \mathrm{aA}$ \\
\hline 13 & $3,12 \mathrm{cA}$ & $3,12 \mathrm{cA}$ & $4,12 \mathrm{bA}$ & $4,12 \mathrm{cA}$ & $6,62 \mathrm{aA}$ & $5,25 \mathrm{cA}$ & $5,87 \mathrm{bA}$ & $5,50 \mathrm{bA}$ \\
\hline 14 & $3,50 \mathrm{bA}$ & $3,50 \mathrm{bA}$ & $5,12 \mathrm{bA}$ & $5,12 \mathrm{bA}$ & $6,75 \mathrm{aA}$ & $6,00 \mathrm{bA}$ & $7,12 \mathrm{aA}$ & $6,12 \mathrm{bA}$ \\
\hline 15 & $3,62 \mathrm{bA}$ & $3,62 \mathrm{bA}$ & $5,37 \mathrm{bA}$ & $5,12 \mathrm{bA}$ & $6,50 \mathrm{aA}$ & $6,75 \mathrm{bA}$ & $6,87 \mathrm{aA}$ & $7,37 \mathrm{aA}$ \\
\hline
\end{tabular}

As médias seguidas da mesma letra minúscula na coluna (substrato), e maiúsculas nas linhas (adubação), não diferem entre si a $5 \%$ de probabilidade pelo teste de Scott-Knott.

Substratos: 1 (terra-T), 2 (esterco-E), 3 (areia-A), 4 (casca de arroz carbonizada-CAC), $5(\mathrm{~T}+\mathrm{E}), 6(\mathrm{~T}+\mathrm{A}), 7(\mathrm{~T}+\mathrm{CAC}), 8(\mathrm{E}+\mathrm{A}), 9(\mathrm{E}+\mathrm{CAC}), 10$ $(\mathrm{A}+\mathrm{CAC}), 11(\mathrm{~T}+\mathrm{E}+\mathrm{A}), 12(\mathrm{~T}+\mathrm{E}+\mathrm{CAC}), 13(\mathrm{~T}+\mathrm{A}+\mathrm{CAC}), 14(\mathrm{E}+\mathrm{A}+\mathrm{CAC}) \mathrm{e} 15(\mathrm{~T}+\mathrm{E}+\mathrm{A}+\mathrm{CAC})$.

*Means followed by the same small letter in columns (substrate) and capital letter in lines (fertilization) did not differ by Scott-Knott test at 5\%.

Substrates: 1 (soil-T), 2 (manure-E), 3 (sand-A), 4 (carbonized rice husk-CAC), $5(\mathrm{~T}+\mathrm{E}), 6(\mathrm{~T}+\mathrm{A}), 7(\mathrm{~T}+\mathrm{CAC}), 8(\mathrm{E}+\mathrm{A}), 9(\mathrm{E}+\mathrm{CAC}), 10(\mathrm{~A}+$ $\mathrm{CAC}), 11(\mathrm{~T}+\mathrm{E}+\mathrm{A}), 12(\mathrm{~T}+\mathrm{E}+\mathrm{CAC}), 13(\mathrm{~T}+\mathrm{A}+\mathrm{CAC}), 14(\mathrm{E}+\mathrm{A}+\mathrm{CAC})$ e $15(\mathrm{~T}+\mathrm{E}+\mathrm{A}+\mathrm{CAC})$ 
análise, aos nove meses, além dos substratos $2(\mathrm{E})$ e $8(\mathrm{E}+$ A), os substratos $11(\mathrm{~T}+\mathrm{E}+\mathrm{A}), 13(\mathrm{~T}+\mathrm{A}+\mathrm{CAC}), 14(\mathrm{E}$ $+\mathrm{A}+\mathrm{CAC})$ e $15(\mathrm{~T}+\mathrm{E}+\mathrm{A}+\mathrm{CAC})$ proporcionaram formação de maior número de folhas. Ao final do experimento, aos 12 meses, outros substratos também proporcionaram formação de maior número de folhas, de modo geral, os que tinham na sua composição esterco bovino. O efeito positivo da adubação foi observado no substrato 1 (terra), em todas as avaliações. Ao contrário, para as plantas cultivadas no substrato 9 (esterco + casca de arroz carbonizada), o desenvolvimento foi melhor sem a adição de adubo na ultima época de avaliação.
O parâmetro diâmetro do caule apresentou interação significativa entre os substratos e a aplicação de adubação fosfatada aos 3, 9 e 12 meses. Foram realizados os desdobramentos para os dois itens testados, e as médias para o diâmetro do caule formado em função dos tratamentos encontram-se na Tabela 4.

Os substratos que proporcionaram diferenças significativas para o diâmetro da planta aos três meses foram o 2 (E, com e sem adubação), 9 (E + CAC), 12 (T + E + CAC), $14(\mathrm{E}+\mathrm{A}+\mathrm{CAC})$ e $15(\mathrm{~T}+\mathrm{E}+\mathrm{A}+\mathrm{CAC})$, sem adubação fosfatada. Aos seis e nove meses, os maiores diâmetros foram obtidos nas plantas cultivadas em substrato consti-

Tabela 4. Médias de diâmetro do caule $(\mathrm{cm})$ de palmeira ráfia cultivadas em diferentes substratos com e sem adição de adubação fosfatada. Avaliações: aos $3\left(1^{\mathrm{a}}\right), 6\left(2^{\mathrm{a}}\right), 9\left(3^{\mathrm{a}}\right)$ e12 $\left(4^{\mathrm{a}}\right)$ meses.

Table 4. Means values of stem diameter of Lady-palm cultivated in different substrates with or without phosphate fertilization. Evaluations: $3\left(1^{\text {st }}\right) ; 6\left(2^{\text {nd }}\right) ; 9\left(3^{\text {rd }}\right)$ and $12\left(4^{\text {th }}\right)$ months.

\begin{tabular}{|c|c|c|c|c|c|c|c|}
\hline \multirow{3}{*}{ Substrato } & \multirow{2}{*}{\multicolumn{2}{|c|}{$\frac{1^{\mathrm{a}} \text { avaliação }}{\text { Adubação }}$}} & \multirow{3}{*}{$2^{\mathrm{a}}$ avaliação } & \multirow{2}{*}{\multicolumn{2}{|c|}{$\frac{3^{\mathrm{a}} \text { avaliação }}{\text { Adubação }}$}} & \multirow{2}{*}{\multicolumn{2}{|c|}{$\frac{4^{\mathrm{a}} \text { avaliação }}{\text { Adubação }}$}} \\
\hline & & & & & & & \\
\hline & Com & Sem & & Com & Sem & Com & Sem \\
\hline 1 & $0,34 \mathrm{bA}$ & $0,25 \mathrm{bB}$ & $0,35 c$ & $0,45 \mathrm{cA}$ & $0,23 \mathrm{~dB}$ & $0,51 \mathrm{bA}$ & $0,23 \mathrm{~dB}$ \\
\hline 2 & $0,46 \mathrm{aA}$ & $0,42 \mathrm{aA}$ & $0,65 \mathrm{a}$ & $0,77 \mathrm{aA}$ & $0,84 \mathrm{aA}$ & $0,86 \mathrm{aA}$ & $1,04 \mathrm{aA}$ \\
\hline 3 & $0,28 \mathrm{cA}$ & $0,28 \mathrm{bA}$ & $0,30 \mathrm{c}$ & $0,29 \mathrm{dA}$ & $0,32 \mathrm{dA}$ & $0,32 \mathrm{cA}$ & $0,38 \mathrm{dA}$ \\
\hline 4 & $0,26 \mathrm{cA}$ & $0,30 \mathrm{bA}$ & $0,32 \mathrm{c}$ & $0,44 \mathrm{cA}$ & $0,40 \mathrm{cA}$ & $0,52 \mathrm{bA}$ & $0,51 \mathrm{cA}$ \\
\hline 5 & $0,32 \mathrm{cA}$ & $0,31 \mathrm{bA}$ & $0,44 \mathrm{~b}$ & $0,55 \mathrm{bA}$ & $0,55 \mathrm{bA}$ & $0,84 \mathrm{aA}$ & $0,68 \mathrm{bA}$ \\
\hline 6 & $0,27 \mathrm{cA}$ & $0,24 \mathrm{bA}$ & $0,33 c$ & $0,39 \mathrm{cA}$ & $0,33 \mathrm{dA}$ & $0,44 \mathrm{cA}$ & $0,39 \mathrm{dA}$ \\
\hline 7 & $0,30 \mathrm{cA}$ & $0,30 \mathrm{bA}$ & $0,38 \mathrm{c}$ & $0,51 \mathrm{cA}$ & $0,44 \mathrm{cA}$ & $0,64 \mathrm{aA}$ & $0,48 \mathrm{cA}$ \\
\hline 8 & $0,35 \mathrm{bA}$ & $0,31 \mathrm{bA}$ & $0,45 b$ & $0,65 \mathrm{bA}$ & $0,55 \mathrm{bA}$ & $0,71 \mathrm{aA}$ & $0,69 \mathrm{bA}$ \\
\hline 9 & $0,33 \mathrm{cB}$ & $0,40 \mathrm{aA}$ & $0,47 b$ & $0,55 \mathrm{bA}$ & $0,64 \mathrm{bA}$ & $0,57 \mathrm{bB}$ & $0,80 \mathrm{bA}$ \\
\hline 10 & $0,25 \mathrm{cA}$ & $0,30 \mathrm{bA}$ & $0,32 \mathrm{c}$ & $0,35 \mathrm{dA}$ & $0,43 \mathrm{cA}$ & $0,39 \mathrm{cA}$ & $0,52 \mathrm{cA}$ \\
\hline 11 & $0,38 \mathrm{bA}$ & $0,30 \mathrm{bB}$ & $0,49 b$ & $0,62 \mathrm{bA}$ & $0,56 \mathrm{bA}$ & $0,74 \mathrm{aA}$ & $0,78 \mathrm{bA}$ \\
\hline 12 & $0,33 \mathrm{cA}$ & $0,35 \mathrm{aA}$ & $0,45 b$ & $0,57 \mathrm{bA}$ & $0,63 \mathrm{bA}$ & $0,70 \mathrm{aA}$ & $0,76 \mathrm{bA}$ \\
\hline 13 & $0,30 \mathrm{cA}$ & $0,29 \mathrm{bA}$ & $0,37 \mathrm{c}$ & $0,47 \mathrm{cA}$ & $0,40 \mathrm{cA}$ & $0,52 \mathrm{bA}$ & $0,49 \mathrm{cA}$ \\
\hline 14 & $0,37 \mathrm{bA}$ & $0,36 \mathrm{aA}$ & $0,46 \mathrm{~b}$ & $0,57 \mathrm{bA}$ & $0,56 \mathrm{bA}$ & $0,69 \mathrm{aA}$ & $0,67 \mathrm{bA}$ \\
\hline 15 & $0,35 \mathrm{bA}$ & $0,38 \mathrm{aA}$ & $0,45 b$ & $0,60 \mathrm{bA}$ & $0,59 \mathrm{bA}$ & $0,75 \mathrm{aA}$ & $0,73 \mathrm{bA}$ \\
\hline
\end{tabular}

As médias seguidas da mesma letra minúscula na coluna (substrato), e maiúsculas nas linhas (adubação), não diferem entre si a 5\% de probabilidade pelo teste de Scott-Knott.

Substratos: 1 (terra-T), 2 (esterco-E), 3 (areia-A), 4 (casca de arroz carbonizada-CAC), $5(\mathrm{~T}+\mathrm{E}), 6(\mathrm{~T}+\mathrm{A}), 7(\mathrm{~T}+\mathrm{CAC}), 8(\mathrm{E}+\mathrm{A}), 9(\mathrm{E}+\mathrm{CAC}), 10$ $(\mathrm{A}+\mathrm{CAC}), 11(\mathrm{~T}+\mathrm{E}+\mathrm{A}), 12(\mathrm{~T}+\mathrm{E}+\mathrm{CAC}), 13(\mathrm{~T}+\mathrm{A}+\mathrm{CAC}), 14(\mathrm{E}+\mathrm{A}+\mathrm{CAC}) \mathrm{e} 15(\mathrm{~T}+\mathrm{E}+\mathrm{A}+\mathrm{CAC})$.

*Means followed by the same small letter in columns (substrate) and capital letter in lines (fertilization) did not differ by Scott-Knott test at $5 \%$. Substrates: 1 (soil-T), 2 (manure-E), 3 (sand-A), 4 (carbonized rice husk-CAC), $5(\mathrm{~T}+\mathrm{E}), 6(\mathrm{~T}+\mathrm{A}), 7(\mathrm{~T}+\mathrm{CAC}), 8(\mathrm{E}+\mathrm{A}), 9(\mathrm{E}+\mathrm{CAC}), 10(\mathrm{~A}+$ $\mathrm{CAC}), 11(\mathrm{~T}+\mathrm{E}+\mathrm{A}), 12(\mathrm{~T}+\mathrm{E}+\mathrm{CAC}), 13(\mathrm{~T}+\mathrm{A}+\mathrm{CAC}), 14(\mathrm{E}+\mathrm{A}+\mathrm{CAC}) \mathrm{e} 15(\mathrm{~T}+\mathrm{E}+\mathrm{A}+\mathrm{CAC})$.

tuído apenas de esterco. Na última análise do experimento, realizada aos 12 meses, apresentaram maiores diâmetros os substratos 2 (E) com ou sem adubação, e os substratos 5 (T $+\mathrm{E}), 7(\mathrm{~T}+\mathrm{CAC}), 8(\mathrm{E}+\mathrm{A}), 11(\mathrm{~T}+\mathrm{E}+\mathrm{A}), 12(\mathrm{~T}+\mathrm{E}+$ $\mathrm{CAC}), 14(\mathrm{E}+\mathrm{A}+\mathrm{CAC})$ e $15(\mathrm{~T}+\mathrm{E}+\mathrm{A}+\mathrm{CAC})$, quando acrescidos de adubação fosfatada, porém, a adição do adubo não proporcionou diferença significativa (Tabela 4).

O efeito da adubação foi observado nos substratos 1 (T), $9(\mathrm{E}+\mathrm{CAC})$ e $11(\mathrm{~T}+\mathrm{E}+\mathrm{A})$, e para plantas cultivadas no substrato 1 (T), o maior diâmetro do caule foi observado com a aplicação de adubo. Já para as plantas cultivadas no substrato $9(\mathrm{E}+\mathrm{CAC})$, houve aumento no diâmetro do caule das plantas aos três e 12 meses, quando elas não receberam adubação. Os substratos que apresentaram matéria orgânica em sua composição proporcionaram formação de plantas com maior diâmetro de caule, assim como observado nos parâmetros altura de plantas e número de folhas.

A formação de brotações é bastante desejável, pois a palmeira-ráfia é comercializada em função do número de hastes que a muda apresenta. Nesse experimento, apenas ocorreram brotações nas plantas cultivadas nos substratos $2(\mathrm{E})$ e $11(\mathrm{~T}+\mathrm{E}+\mathrm{A})$ durante a última avaliação (aos 12 meses), com uma média de 0,37 e 0,12 brotos por planta, para ambos os substratos. Os substratos com diferentes formulações proporcionam desenvolvimento semelhante nas plantas de palmeira-ráfia, quando avaliadas as características altura, número de folhas, diâmetro de caule e número de brotos. Entretanto, observou-se que os substratos que proporcionaram melhores incrementos têm em sua composição o esterco, evidenciando o efeito da matéria orgâ- 
nica no crescimento da espécie, efeito também observado por McKAMEY (1989), que indica como essencial o uso de matéria orgânica na composição de substratos para cultivo de $R$. excelsa. A efetividade do esterco como substrato para outras culturas também já foi observada por ANDRADE NETO (1998), que verificou maior desenvolvimento de plantas de café utilizando esterco de curral na proporção de $80 \%$ do substrato e adubação com osmocote. A eficiência do substrato esterco pode ser atribuída ao teor elevado de macro e micronutrientes como $\mathrm{N}, \mathrm{P}, \mathrm{K}, \mathrm{B}$ e $\mathrm{Cu}$, como pode ser observado na Tabela 1, que apresenta os resultados da análise química dos substratos utilizados.

$\mathrm{O}$ uso de terra em proporções maiores na composição de substratos foi indicado por AGUIAR et al. (1996) e AGUIAR et al. (2002) para cultivo de Geonoma schottiana e Euterpe edulis, respectivamente, e também por BOVI (1994) no cultivo de Euterpe oleraceae. Mas, ao contrário do sugerido por esses autores, o uso de terra não proporcionou bons resultados para o desenvolvimento de $R$. excelsa.

Pelos resultados obtidos nesse experimento, não se observou influência significativa quando da aplicação de adubação fosfatada nos substratos. Verificou-se em todos os parâmetros analisados a superioridade do substrato 2 (esterco) em relação aos demais tratamentos. Os substratos $11(\mathrm{~T}+$ $\mathrm{E}+\mathrm{A}), 12(\mathrm{~T}+\mathrm{E}+\mathrm{CAC})$ e $15(\mathrm{~T}+\mathrm{E}+\mathrm{A}+\mathrm{CAC})$, além de proporcionarem formação de mudas com alta qualidade, equivalentes às obtidas no substrato $2(\mathrm{E})$, são mais viáveis para uso hortícola e de fácil aquisição para produtores ou viveiristas, podendo assim ser considerados adequados para a produção de mudas de palmeira-ráfia.

\section{CONCLUSÕES}

Nas condições de realização desse experimento, sugerem-se como substratos para o cultivo de mudas jovens de palmeira-ráfia as composições de substratos: terra + esterco + areia, terra + esterco + casca de arroz carbonizada e terra + esterco + areia + casca de arroz carbonizada.

Não há necessidade de adubação fosfatada no substrato.

\section{REFERÊNCIAS}

AGUIAR, F. F. A.; KANASHIRO, S.; BARBEDO, C. J. Efeito da calagem e da adubação mineral e orgânica na formação de mudas de Geonoma schottiana Mart. Revista Brasileira de Horticultura Ornamental, Campinas, v. 2, n. 1, p. 33-36, 1996.

AGUIAR, F. F. A.; SCHAEFER, S. M.; LOPES, E. A.; TOLEDO, C. B. Produção de mudas de palmito-juçara $E \boldsymbol{u}$ terpe edulis Mart. São Paulo: Instituto de Botânica, 2002. $16 \mathrm{p}$.

ANDRADE NETO, A. Avaliação de substratos alternativos e tipos de adubação para a produção de mudas de cafeeiro (Coffea arabica L.) em tubetes. Lavras: Universidade Federal de Lavras, 1998. 65 p. Dissertação de Mestrado em Fitotecnia.
BLOMBERY, A.; ROOD, T. Palms: an informative practical guide to palms of the world; their cultivation, care and landscape use. London: Angus \& Robertson, 1982. 201 p.

BOVI, M. L. A. Nursery growth of Euterpe oleracea as a function of substrate and container size. Acta Horticulturae, Amsterdam, v. 360, p. 195-209, 1994.

BRASIL. Ministério da Agricultura. Normas climatológicas: 1961-1990. Brasília: EMBRAPA/DNMET, 1992. 84p.

BROSCHAT, T. K.; MEEROW, A. W. Palm nutrition guide. Gainesville: University of Florida Extension Circular SS-ORH-02, 1990.

CUNHA, A.M.; CUNHA, G.M.; SARMENTO, R.A.; CUNHA, G.M.; AMARAL, J.F.T. Efeito de diferentes substratos sobre o desenvolvimento de mudas de Acacia sp. Revista Árvore, Viçosa, v.30, n.2, p.207-214, 2006.

DEMATTÊ, J. B. I.; GRAZIANO, T. T.; VOLPE, C. A. PERECIN, D.; DEMATTÊ, M. E. S. P. Interaction between substrates and irrigation on early development of Rhapis excelsa (LADY PALM). Acta Horticulturae, Amsterdam, v. 360, p. 211-216, 1994.

DOMINGUES, R. C. Espécies ornamentais. Natureza, São Paulo, v. 5, n. 8, p. 14-18, 1995.

FERREIRA, D. F. Análises estatísticas por meio do Sisvar para Windows versão 4. 0. In: REUNIÃO ANUAL DA REGIÃO BRASILEIRA DA SOCIEDADE INTERNACIONAL DE BIOMETRIA, 45., Piracicaba, 2000, São Carlos. Anais... São Carlos: UFSCar, 2000. p. 255-258.

LORENZI, H.; SOUZA, H. M.; COSTA, J. T. M.; CERQUEIRA, L. S. C.; FERREIRA, E. Palmeiras brasileiras e exóticas e cultivadas. Nova Odessa: Instituto Plantarum, 2004. $416 \mathrm{p}$.

LUZ, P.B.; PAIVA, P.D.O.; TAVARES, A.R. Effect of foliar and substrate fertilization on lady palm seedling growth and development. Journal of Plant Nutrition, London, v.31: p. 1311-1318, 2008.

McCURRACH, J. C. Palms of the world. New York: Harper and Bros, 1960. 290 p.

McKAMEY, L. RHAPIS PALMS Culitivated Species \& Varieties Culture and Care of the "Ladies". Principes, Miami, v. 33, n. 3, p. 129-139, 1989.

SONNEVELD, C.; VAN DEN, J.; VAN DIJK, P. A. Analysis of growing media by means of a 1:1,5 volume extract. Communications in Soil Science and Plant Analysis, New York, v. 5, n. 30, p. 183-202, 1974. 